\title{
Shape-shifting MRI probes
}

Tiny, environmentally responsive, magnetic resonance active nanostructures serve as shape-changing MRI sensors.

Magnetic resonance imaging (MRI) contrast agents, for all their value as imaging probes in biology and medicine, have some severe limitations: their signals are difficult to quantify accurately, and they lack multiplexing capabilities. Researchers at the US National Institute of Neurological Disorders and Stroke hope that they can potentially help overcome the shortcomings of current contrast agents, using microengineering.

Gary Zabow and his colleagues recently introduced a new kind of microengineered, environmentally responsive sensor as an MRI probe. These shape-changing geometrically encoded magnetic (GEM) sensors consist of two microfabricated disks made from a magnetic material-iron or nickelsandwiching a hydrogel that responds to the local environment. This tiny device is barely more than a micrometer in size. In the presence of an environmental stimulus, the hydrogel can swell or shrink, changing the distance between the magnetic disks and thus the magnetic resonance frequency shift.

Zabow's team showed that GEM sensors consisting of a hydrogel responsive to $\mathrm{pH}$ swell under basic conditions and shrink under acidic conditions, thereby serving as a sensitive, MRI-active reporter. Using an array of sensors, the team tracked the acidification of the medium surrounding Madin-Darby canine kidney cells in an enclosed container due to carbon dioxide production. As expected, the researchers found that acidification and cell death were accelerated at elevated temperature.

Unlike traditional MRI contrast agents, GEM sensors produce a frequency readout that is independent of concentration. Though the sensor response time does depend on hydrogel swelling or shrinking,
Zabow and his colleagues estimate that this delay is less than a second. A notable benefit of these GEM sensors is that it is relatively straightforward to fabricate spectrally isolated sensors for 'multicolor' imaging, simply by changing the size of the disks or the hydrogel material.

Though the work is preliminary in terms of in vivo applications, Zabow emphasizes that biological research has been a major driver in developing GEM sensors. "The sensor design is intended to make it adaptable to measure a variety of physiological and environmental conditions which might include biologically relevant variables such as $\mathrm{pH}$, temperature, glucose, calcium, other analytes and enzymes, etc." he says.

\section{Allison Doerr}

\section{RESEARCH PAPERS}

Zabow, G. et al. Shape-changing magnetic assemblies as high-sensitivity NMR-readable nanoprobes. Nature 520, 73-77 (2015). 